\title{
Time-Sensitive Therapeutic Interventions at Diagnosis of Sepsis: Should Guidelines Be Confined to High-Level Evidence?
}

\author{
Lavi Oud
}

\section{To the Editor}

Sepsis (formerly severe sepsis [1]) is increasingly recognized as a global health threat and is associated with substantial morbidity and mortality [2]. Sepsis is currently defined as life-threatening organ dysfunction due to dysregulated host response to infection [1], and is considered a medical emergency $[2,3]$.

Following the demonstrated outcome benefits of timely initiation of antimicrobial therapy and circulatory support in a small randomized sepsis trial [4], the Surviving Sepsis Campaign (SSC), a collaborative initiative by professional societies, has introduced serially updated guidelines on time-sensitive care bundles for sepsis including, among other elements, initiation of broad-spectrum antibiotics within $1 \mathrm{~h}$ of patient's presentation (e.g. time of triage in the Emergency Department) and initial provision of at least $30 \mathrm{~mL} / \mathrm{kg}$ of intravenous fluids in patients with sepsis-induced hypoperfusion (defined as hypotension and/or serum lactate level $\geq 4 \mathrm{mmol} / \mathrm{L}$ ) [3]. Increasing compliance with bundle-based care was associated with improved patient outcomes [5].

More recently, the SSC has updated its guidelines, introducing a 1-h bundle where the abovementioned fluid resuscitation was also to be initiated within $1 \mathrm{~h}$ of presentation [6]. This update brought forth progressively increasing opposition to the advocated bundled care and, specifically, to both early antimicrobial therapy [7] and the recommended fluid administration to all those with sepsis-induced hypoperfusion [8]. The concerns raised about the latter two interventions focused predominantly on limitations of supporting evidence and risks of adverse impact of such broad guidance for treatment of a syndromic condition, with ongoing challenges in its precise diagnosis.

The specific arguments brought by the opponents of administration of timed antimicrobial therapy in patients considered septic include the following. 1) Even among patients with

Manuscript submitted May 2, 2019, accepted May 23, 2019

Division of Pulmonary and Critical Care Medicine, Department of Internal Medicine, Texas Tech University Health Sciences Center at the Permian Basin, 701 W. 5th St., Odessa, TX 79763, USA.Email: lavi.oud@ttuhsc.edu

doi: https://doi.org/10.14740/jocmr3866 a diagnosis of septic shock, a considerable proportion (11.6\%) was found to have non-infectious etiology after 24 following ICU admission [9]. 2) An overly broad mandate for administration of antibiotics will increase antimicrobial resistance, especially if clinicians will feel pressured to administer antibiotics to patients who are not infected [7]. 3) Previous studies showing increased risk of death with hourly delays in antibiotic therapy were retrospective with expected uncontrolled confounders [7].

However, other perspectives on the contemporary data on sepsis care should be considered by clinicians when contemplating use and initial timing of antibiotics in patients suspected to be septic. 1) The large number of observational studies showing increased risk of death with progressive delay of initial antimicrobial therapy in septic patients $[10,11]$ do not represent a therapeutic equipoise and thus a randomized trial on timing of initial antibiotics in sepsis is unlikely due to ethical considerations, while early administration of antibiotics in severe bacterial and fungal infection is biologically plausible; thus, it is unlikely that higher level of evidence for timing of initial antibiotics in suspected sepsis is forthcoming. 2) Although unnecessary, prolonged antimicrobial therapy will undoubtedly increase development of antimicrobial resistance, there are no reports demonstrating the association of an empiric single initial dose of antibiotics (or that for the first 24 - $48 \mathrm{~h}$ ) for suspected sepsis or septic shock with increasing antimicrobial resistance at institutional or population-level. Crucially, no study to date has demonstrated that the risk of initial antimicrobial therapy in a patient suspected to have sepsis or septic shock, but shown later to have no infectious etiology outweighs the risk of delay of proper antimicrobial therapy in a patient with suspected sepsis/septic shock, with ultimately confirmed infection. 3) There are no reports to suggest that data definitely confirming or excluding an infectious cause in a patient with suspected sepsis/septic shock can be routinely expected within $3-6 \mathrm{~h}$ after initial presentation. It is, moreover, inconceivable that a clinician will delay initial antimicrobial therapy for several hours once a patient with new life-threatening organ dysfunction is suspected to have a potentially treatable infectious etiology. Importantly, even in the setting of a clinical trial of septic shock, with heightened pre-enrollment scrutiny, a specific site of infection could not be identified in $12.7 \%$ of enrolled patients [12] and microbial etiology could not be demonstrated in $36 \%$ [13].

When addressing use of initial weight-based intravenous 
fluid resuscitation in patients considered to have sepsis-induced hypoperfusion, the key objections to the SSC guidance brought fore are: 1) Lack of data from animal experiments or clinical trials to support the recommended weight-based fluid regimen and its uniform use in hypotensive and/or hyperlactatemic patients [8], coupled with studies showing lack of beneficial effect of this part of bundled care [10]. 2) There is consistent evidence of increased mortality among septic patients with high fluid balance $[14,15] .3)$ Only $50 \%$ of septic patients presenting to the Emergency Department are fluid-responsive (e.g. demonstrating increase in cardiac output or stroke volume with fluid administration) [16]. 4) Aggressive administration of intravenous fluids may increase the risk of adverse events (e.g. respiratory failure), especially among high-risk populations (e.g. congestive heart failure, chronic renal failure).

Nevertheless, several pragmatic issues must be considered when appraising the application of the fluid-related SSC guideline. 1) Both the duration and severity of hypotension are strongly associated in a graded fashion with increased morbidity and mortality among septic patients [17]. On the other hand, although no experimental data support the specific weightbased fluid recommendation, there are similarly no data to support exclusive or prompt use of vasopressors as initial or primary intervention in patients with sepsis-induced hypoperfusion. 2) The data on the adverse risk of high fluid balance in critically ill patients were never demonstrated about the initial weight-based fluid administration per SSC guideline. On the other hand, a recent population-based performance improvement study of sepsis care demonstrated that improved hospital mortality with increasing compliance with bundled care was mediated by increased administration of intravenous fluids in patients with congestive heart failure and renal failure [18], the very groups considered at increased risk from the broad recommendation for initial fluid administration by the SSC. 3) In a recent multicenter cohort study of sepsis and septic shock, the lowest adjusted risk of death was observed among those receiving intravenous fluids within $30 \mathrm{~min}$ of sepsis identification and with a volume of $20-35 \mathrm{~mL} / \mathrm{kg}$ [19]. 4) Physical examination is inadequate to accurately determine intravascular volume [20] or cardiac output (e.g. low, normal, or high) [21]. However, expertise in use of noninvasive tools to determine fluid responsiveness (e.g. ultrasound) is generally not available at point-of-care outside the ICU, thus precluding routine assessment of fluid responsiveness at initial presentation.

In summary, while considerable concerns were raised about the SSC guideline on the initial time-sensitive antimicrobial therapy and fluid administration in patients with suspected sepsis, no pragmatically safe and data-supported alternatives have been offered by opponents, although acknowledging that sepsis is a medical emergency. In addition, no study to date demonstrated harm from any of the elements of the SSC initial care bundle.

Thus, until point-of-care diagnostic tools become available to conclusively confirm or exclude a treatable infection in patients with suspected sepsis, prompt initiation of targeted broad-spectrum antibiotics in these patients appears prudent, while clinicians should continue to carefully appraise new clinical data confirming or excluding an infectious etiology, to allow early discontinuation of antibiotics if a non-infectious cause of organ dysfunction becomes apparent. Similarly, pending data from an ongoing study on the relative role of early fluids vs. vasopressors in sepsis-induced hypotension [22] and until point-of-care tools to determine fluid-responsiveness among hemodynamically unstable septic patients become routinely available, the current fluid recommendation by SSC appears reasonable to consider, with the exception of patients with evidence of cardiogenic or hypervolemic pulmonary edema.

\section{Acknowledgments}

None.

\section{Financial Disclosure}

None.

\section{Conflict of Interest}

None.

\section{Informed Consent}

Not applicable.

\section{Author Contributions}

Not applicable.

\section{References}

1. Singer M, Deutschman CS, Seymour CW, Shankar-Hari M, Annane D, Bauer M, Bellomo R, et al. The third international consensus definitions for sepsis and septic shock (Sepsis-3). JAMA. 2016;315(8):801-810.

2. Sepsis. World Health Organization. Available from: https://www.who.int/news-room/fact-sheets/detail/sepsis.

3. Rhodes A, Evans LE, Alhazzani W, Levy MM, Antonelli M, Ferrer R, Kumar A, et al. Surviving sepsis campaign: international guidelines for management of sepsis and septic shock: 2016. Crit Care Med. 2017;45(3):486-552.

4. Rivers E, Nguyen B, Havstad S, Ressler J, Muzzin A, Knoblich B, Peterson E, et al. Early goal-directed therapy in the treatment of severe sepsis and septic shock. N Engl J Med. 2001;345(19):1368-1377.

5. Levy MM, Rhodes A, Phillips GS, Townsend SR, Schorr CA, Beale R, Osborn T, et al. Surviving Sepsis Campaign: association between performance metrics and outcomes in a 7.5-year study. Crit Care Med. 2015;43(1):3-12.

6. Levy MM, Evans LE, Rhodes A. The surviving sepsis campaign bundle: 2018 update. Crit Care Med. 
2018;46(6):997-1000.

7. Singer M. Antibiotics for sepsis: does each hour really count, or is it incestuous amplification? Am J Respir Crit Care Med. 2017;196(7):800-802.

8. Marik PE, Farkas JD, Spiegel R, Weingart S, collaborating authors. POINT: Should the Surviving Sepsis Campaign Guidelines Be Retired? Yes. Chest. 2019;155(1):12-14.

9. Contou D, Roux D, Jochmans S, Coudroy R, Guerot E, Grimaldi D, Ricome S, et al. Septic shock with no diagnosis at 24 hours: a pragmatic multicenter prospective cohort study. Crit Care. 2016;20(1):360.

10. Seymour CW, Gesten F, Prescott HC, Friedrich ME, Iwashyna TJ, Phillips GS, Lemeshow S, et al. Time to treatment and mortality during mandated emergency care for sepsis. N Engl J Med. 2017;376(23):2235-2244.

11. Liu VX, Fielding-Singh V, Greene JD, Baker JM, Iwashyna TJ, Bhattacharya J, Escobar GJ. The timing of early antibiotics and hospital mortality in sepsis. Am J Respir Crit Care Med. 2017;196(7):856-863.

12. ProCESS Investigators, Yealy DM, Kellum JA, Huang DT, Barnato AE, Weissfeld LA, Pike F, et al. A randomized trial of protocol-based care for early septic shock. N Engl J Med. 2014;370(18):1683-1693.

13. ARISE Investigators; ANZICS Clinical Trials Group, Peake SL, Delaney A, Bailey M, Bellomo R, Cameron $\mathrm{PA}$, et al. Goal-directed resuscitation for patients with early septic shock. N Engl J Med. 2014;371(16):14961506.

14. Boyd JH, Forbes J, Nakada TA, Walley KR, Russell JA. Fluid resuscitation in septic shock: a positive fluid balance and elevated central venous pressure are associated with increased mortality. Crit Care Med. 2011;39(2):259265.

15. Sakr Y, Rubatto Birri PN, Kotfis K, Nanchal R, Shah B, Kluge S, Schroeder ME, et al. Higher fluid balance increases the risk of death from sepsis: results from a large international audit. Crit Care Med. 2017;45(3):386-394.

16. Smith B, Phillips R, Madigan V, West M. Decreased mortality, morbidity and emergency transport in septic shock: a new protocol based on advanced noninvasive haemodynamics and early antibiotics. Crit Care Med. 2013;40(12):1-328.

17. Maheshwari K, Nathanson BH, Munson SH, Khangulov V, Stevens M, Badani H, Khanna AK, et al. The relationship between ICU hypotension and in-hospital mortality and morbidity in septic patients. Intensive Care Med. 2018;44(6):857-867.

18. Liu VX, Morehouse JW, Marelich GP, Soule J, Russell T, Skeath M, Adams C, et al. Multicenter Implementation of a Treatment Bundle for Patients with Sepsis and Intermediate Lactate Values. Am J Respir Crit Care Med. 2016;193(11):1264-1270

19. Leisman DE, Goldman C, Doerfler ME, Masick KD, Dries S, Hamilton E, Narasimhan M, et al. Patterns and outcomes associated with timeliness of initial crystalloid resuscitation in a prospective sepsis and septic shock cohort. Crit Care Med. 2017;45(10):1596-1606.

20. Saugel B, Ringmaier S, Holzapfel K, Schuster T, Phillip V, Schmid RM, Huber W. Physical examination, central venous pressure, and chest radiography for the prediction of transpulmonary thermodilution-derived hemodynamic parameters in critically ill patients: a prospective trial. J Crit Care. 2011;26(4):402-410.

21. Hiemstra B, Koster G, Wiersema R, Hummel YM, van der Harst P, Snieder H, Eck RJ, et al. The diagnostic accuracy of clinical examination for estimating cardiac index in critically ill patients: the Simple Intensive Care Studies-I. Intensive Care Med. 2019;45(2):190-200.

22. Crystalloid Liberal of Vasopressors Early Resuscitation in Sepsis (CLOVERS). ClinicalTrials.gov NCT03434028. Available from: https://clinicaltrials.gov/ct2/show/NCT0 3434028. 